\title{
A Novel Frequency Estimation of Sinusoid by iterative interpolation DFT algorithm
}

\author{
Chao Huang ${ }^{1,2, a}$, Jidong Suo ${ }^{1, b}$ and Guiming Shi, c \\ ${ }^{1}$ Institute of Information and Technology, Dalian Maritime University, Dalian 116026, China; \\ ${ }^{2}$ City Institute of Dalian University of Technology, Dalian 116600, China; \\ ${ }^{3}$ Dalian Institute of Science and Technology, Dalian 116052, China; \\ a39273591@qq.com, bsjddmu@sina.com, cshiguiming1983@163.com
} Keywords: Frequency estimation, polarity estimation, Discrete Fourier transform, Iterative
interpolation, Cramer-Rao low bound.

\begin{abstract}
The frequency estimation of sinusoidal signal based on discrete fourier transform (DFT) is investigated, which performance is caused to decline due to the error of interpolation direction when the signal frequency is close to quantized frequency of DFT. To solve this problem, a new algorithm without weighting window is proposed. Firstly, a coarse frequency estimation in DFT spectrum is acquired, then a high-accuracy frequency near the coarse frequency by iterative interpolation is estimated. The simulation results show that errors caused by a mistaken location of the spectral line can be significantly reduced and the algorithm is simple and has good performance in mean square error and accuracy.
\end{abstract}

\section{Introduction}

The frequency estimation of sinusoidal signal which is submerged in the noise, whether in theory or in practice, is a topic of great research value. Many scholars at home and abroad have done a lot of research on this problem, and put forward many methods in time domain and in frequency domain. Because of FFT fast algorithms, the spectral analysis method based on DFT is especially suitable for real-time signal processing. Unfortunately,their estimation accuracy is affected by two drawbacks:the spectral leakage and the picket-fence, even if there is no noise, the sinusoidal signal spectrum is leaking when the sampling frequency is not an integer multiple of DFT frequency resolution, the real frequency of signal falls between two discrete FFT spectral lines, resulting in frequency estimation can not meet the accuracy requirements and various interpolation strategy emerged. the frequency estimation of sinusoidal signal based on FFT is currently divided into two processes: rough estimation and precise estimation. Coarse estimation is accomplished by directly observing the maximum FFT amplitude spectrum. The precise estimation based on the line spectrum of sinusoidal signal by some interpolation strategies, can estimate sinusoidal signals accurately.

Rife etal. [1] proposed a frequency estimator based on the modulus of two FFT spectral lines,but the Rife's algorithm can easily lead to errors in the second spectral line which leads to the error of interpolation. To solve the problem,the algorithm with weighting window was investigated. Jain etal. [2] investigated the problem for the rectangle window. Later, it was extended for the Hanning window [3] and for Rife-Vincent class I windows [4]. the same idea with a different explanation was developed by Xie and Ding in [5], in which both rectangle window and Hanning window were studied. Similar estimators were explored for Hanning-windowed data[6]. The estimators were further improved and extended for decaying sinusoids or exponentials [7-9]. The Kasier-Bessel and Dolph-Chebyshev windows are known for superior performance in multitone detection[10]. Frequency estimation of the weighted real tones [11].

Because the signal to noise ratio (SNR) is related to the length of the data,however,the weighting window will cause the signal length to be shortened, and then reduce the SNR.

The purpose of this paper is to present an iterative DFT interpola-tion algorithm without weighting window that can accurately estimate frequency in a discrete spectrum and incorrect polarity 
estimation can almost be completely avoided in the proposed algorithm. the remainder of the paper is organized as follows: in section 2 summary of analytic DFT interpolation formulas is briefly described. the analytical expression for the proposed frequency estimator is derived in Section 3. the accuracies of the derived expressions are verified through computer simulations in section 4 . conclusions are given in section 5 .

\section{Theoretical background}

The frequency estimation of discrete sinusoidal signal can be modeled as:

$$
s(n)=a \cos \left(2 \pi f_{0} n T / N+\theta_{0}\right)+v(n) .
$$

where $a$ is amplitude, $f_{0}$ is frequency, $\theta_{0}$ is the phase angle, $T$ is the sampling time interval and $n$ is the sample index, $v(n)$ may be assumed to be additive white gaussian noise with zero mean and a variance of $\sigma_{n}{ }^{2}$.

Based on the DFT definition, the DFT coefficients $S(k)$ can be computed by

$$
S(k)=\sum_{n=0}^{N-1} s(n) \cdot e^{-j \frac{2 \pi}{N} k n}+V(k), 0 \leq k \leq N-1 .
$$

$V(k)$ is the DFT of $v(n)$.in view of the symmetry of real sequences, the negative rate components of DFT spectrum are neglected, and only the front N/2 points of the discrete spectrum are considered. the $S(k)$ can also be described as:

$$
S(k)=\frac{a \cdot \sin \left[\pi\left(k-f_{0} T\right)\right]}{2 \sin \left[\pi\left(k-f_{0} T\right) / N\right]} \cdot e^{j\left[\theta_{0}-\frac{N-1}{N}\left(k-f_{0} T\right) \pi\right]}+V(k), 0 \leq k \leq N / 2-1
$$

Maximum value of $S(k)$ can be noted by approximately:

$$
B_{1}=\max \left|S\left(k_{1}\right)\right|=\frac{N a \cdot \sin [\pi \delta]}{2 \pi \delta},|\delta|<0.5
$$

where $k_{1}$ is the index value at the maximum of $S(k)$ and $k_{1}=\operatorname{int}\left[f_{0} T\right]$,int $[y]$ represents an integer closest to y, $\delta=f_{0} T-k_{1}, B_{1}$ is the maximum of $S(k)$.if $k_{2}$ is the index of the second largest bin of $S(k)$,then $k_{2}=k_{1}+1$ or $k_{2}=k_{1}-1$. the second largest bin can be noted by approximately:

$$
B_{2}=\max \left|S\left(k_{2}\right)\right|=\frac{N a \cdot|\sin [\pi \delta]|}{2 \pi(1-|\delta|)}
$$

the ratio of the two largest magnitudes is expressed as

$$
b=\frac{B_{2}}{B_{1}}=\frac{|\delta|}{1-|\delta|}
$$

according to the ratio of $B_{2}$ to $B_{1}, \hat{\delta}$ can be expressed as

$$
\hat{\delta}= \pm \frac{b}{1+b}
$$

if the index of the second largest bin is $k_{1}+1$, then $\hat{\delta}>0$. if the index of the second largest bin is $k_{1}-1$, then $\hat{\delta}<0$. if signal-to-noise ratio (SNR) is well above the so-called "threshold"[12], the precise frequency estimation can be computed by

$$
\widehat{f}=\left(k_{1}+\widehat{\delta}\right) T
$$

Eq.(7) shows that the $\widehat{\delta}$ depends on the second largest magnitude of $S(k)$. when $\widehat{\delta}$ is near zero, correctly locating the correct spectral line is often difficult because of wideband noise or spectral leakage. as a result, some methods could be applied to solve this problem by weighting window such as Hamming window, Kasier-Bessel windows, Dolph-Chebyshev windows etal. however, it would 
reduce the SNR at the same time. The aim of this paper is to present an iterative DFT interpolation algorithm without weighting window that can accurately estimate frequency in a discrete spectrum.

\section{Proposed algorithm}

In Section2, Eq.(8) shows that the $\hat{f}$ is easy to be affected by the index of the second largest bin. in order to avoid incorrect polarity estimation, the paper proposed a novel algorithm which just need to find the maximum value in a discrete spectrum, the specific steps are as follows:

Step 1: From Eq.(4), acquire the maximum at time index $k_{1}, S\left(k_{1}\right)$.the original frequency must locate in $\left[k_{1}-0.5, k_{1}+0.5\right]$.

Step 2: Apply the parameters obtained from Step 1 to the following fomula (9) as

$$
S\left(k_{1}, r\right)=\sum_{n=0}^{N-1} s(n) \cdot e^{-j \frac{2 \pi}{N}\left(k_{1}+\frac{1}{2 M} r\right) n}+V\left(k_{1}\right)
$$

Where $M$ is an arbitrary natural number except zero, $r$ is an arbitrary integer which locate in $[-M, M]$. maximum value of $S\left(k_{1}, r\right)$ can be noted by approximately:

$$
\max \left|S\left(k_{1}, r\right)\right|=\left|S\left(k_{1}, \hat{r}\right)\right|=\frac{N a \cdot \sin \left[\pi\left(f_{0} T-k_{2}\right)\right]}{2 \pi\left(f_{0} T-k_{2}\right)}
$$

Where $k_{2}=k_{1}+\frac{1}{2 M} \hat{r}, \hat{r} \in[-M, M]$,from Eq.(9) and Eq.(10), We can judge the original frequency must locate in $\left[k_{2}-\frac{1}{4 M}, k_{2}+\frac{1}{4 M}\right]$.

Step 3: Apply the parameters obtained from Step 2 to the following fomula (11) as

$$
S\left(k_{2}, \xi\right)=\sum_{n=0}^{N-1} s(n) \cdot e^{-j \frac{2 \pi}{N}\left(k_{2}+\frac{1}{4 M P} \xi\right) n}+V\left(k_{2}\right)
$$

Where $P$ is an arbitrary natural number except zero, $\xi$ is an arbitrary integer which locate in $[-P, P]$. maximum value of $S\left(k_{2}, \xi\right)$ can be noted by approximately:

$$
\max \left|S\left(k_{2}, \xi\right)\right|=\left|S\left(k_{2}, \hat{\xi}\right)\right|=\frac{N a \cdot \sin \left[\pi\left(f_{0} T-k_{3}\right)\right]}{2 \pi\left(f_{0} T-k_{3}\right)}
$$

Where $k_{3}=k_{2}+\frac{1}{4 M P} \hat{\xi}, \hat{\xi} \in[-P, P]$,from Eq.(11) and Eq.(12), We can judge the original frequency must locate in $\left[k_{3}-\frac{1}{8 M P}, k_{3}+\frac{1}{8 M P}\right]$.

Step 4: Estimate the frequency using the following Eq.(13) as

$$
\widehat{f}=k_{3} T
$$

In theory, with the increase of $M$ or $P$, the frequency estimation is becoming more and more accurate. however, considering the amount of calculation, the $M$ or $P$ should not be too large. morever, if SNR is too low, the largest magnitude may be badly distorted by noise, when the $M$ or $P$ increases to a certian value, the performance of frequency estimation has remained constant.

\section{Simulation results}

In this section the accuracies of expressions (13) are firstly verified by means of computer simulations. Then, the accuracies of the proposed procedure, L\&Z\&M's algorithm [11],the classical Rife's method [12] are compared through both computer simulations and experimental results. It is worth noticing that in [11] it has been shown that the L\&Z\&M's algorithm provides more accurate 
frequency estimates than previous algorithms. as a basis for comparison, the CramerRao bound (CRB) [1] is given by

$$
\sigma_{C R B}^{2}=\frac{3 f_{s}^{2}}{\pi^{2} \cdot N \cdot\left(N^{2}-1\right) \cdot S N R}
$$

Where $\mathrm{N}$ represents the number of samples.the simulation was performed with additional white gaussian noise, Phase was set to zero,The number of samples was 512 and the sampling rate was $1000 \mathrm{~Hz}$.

When the estimated freqency is $250 \mathrm{~Hz}\left(f_{0}=250 \mathrm{~Hz}\right)$, Fig. 1 shows the performance of frequency estimation in different SNR. from the simulation results, the performance of frequency estimation is improved with the increase of $\mathrm{M}$. in practice, the bigger $\mathrm{M}$ or $\mathrm{P}$ has led to a large amount of calculation, so a trade-off must be considered between the performance and the calculation. Fig.2 indicates the performane of the proposed algorith approachs to the CramerRao low bound[1] when the SNR is above zero.

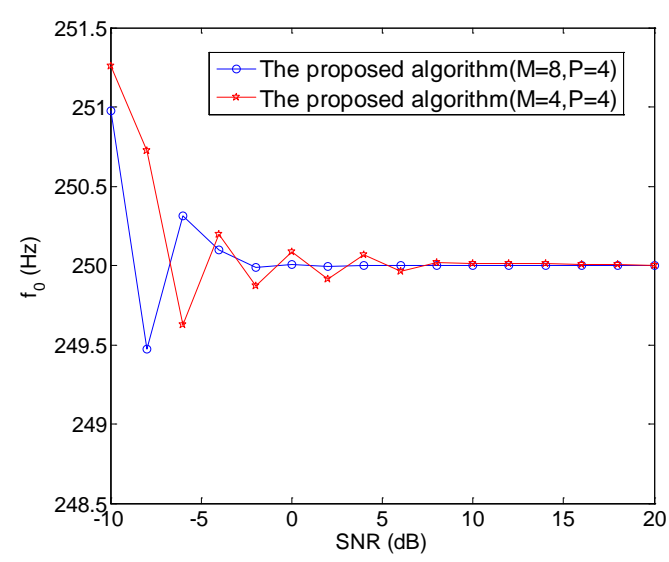

Fig.1. The estimate frequency vs. SNR .

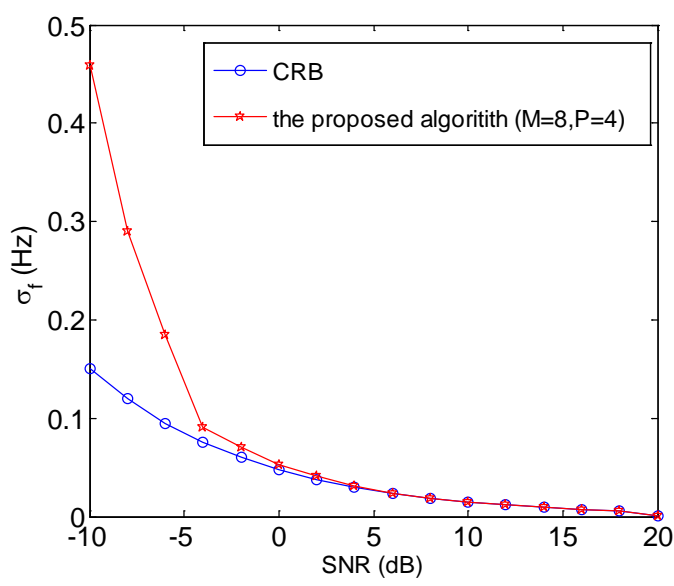

Fig.2. The variances of frequency vs. SNR

Table 1. RMSE of frequency estimation vs. the input signal frequency by using Rife's method, L\&Z\&M's algorithm with weighting Hanning window and The proposed algorithm in this paper (where $M=8, P=4$ ).

\begin{tabular}{|c|c|c|c|c|c|c|c|}
\hline \multirow[t]{2}{*}{$f_{0}(\mathrm{~Hz})$} & \multirow[t]{2}{*}{$\tilde{\delta}$} & \multicolumn{4}{|c|}{$\begin{array}{c}\text { L\&Z\&M’s algorithm } \\
\text { with weighting } \\
\text { Hanning window }\end{array}$} & \multicolumn{2}{|c|}{ The proposed algorithm } \\
\hline & & $\bar{f}_{0}(\mathrm{~Hz})$ & $\sigma_{f}(\mathrm{~Hz})$ & $\bar{f}_{0}(\mathrm{~Hz})$ & $\sigma_{f}(\mathrm{~Hz})$ & $\bar{f}_{0}(\mathrm{~Hz})$ & $\sigma_{f}(\mathrm{~Hz})$ \\
\hline 249.0234 & -0.5 & 249.0485 & 0.01698 & 249.0267 & 0.01697 & 249.0271 & 0.01688 \\
\hline 249.2188 & -0.4 & 249.2430 & 0.01709 & 249.2322 & 0.01699 & 249.2177 & 0.01689 \\
\hline 249.4141 & -0.3 & 249.4542 & 0.01894 & 249.4108 & 0.01715 & 249.4182 & 0.01712 \\
\hline 249.6094 & -0.2 & 249.6402 & 0.09627 & 249.6312 & 0.01692 & 249.6203 & 0.01691 \\
\hline 249.8047 & -0.1 & 249.8237 & 0.1131 & 249.8126 & 0.01734 & 249.8063 & 0.01692 \\
\hline 250.0000 & 0 & 249.9001 & 0.1083 & 249.9758 & 0.01694 & 250.0013 & 0.01697 \\
\hline 250.1953 & 0.1 & 250.2103 & 0.1174 & 250.0958 & 0.01746 & 250.2001 & 0.01689 \\
\hline 250.3906 & 0.2 & 250.3875 & 0.09971 & 250.2879 & 0.01719 & 250.3925 & 0.01698 \\
\hline 250.5859 & 0.3 & 250.5924 & 0.01841 & 250.5968 & 0.01704 & 250.5932 & 0.01693 \\
\hline 250.7813 & 0.4 & 250.7781 & 0.01785 & 250.8003 & 0.01754 & 250.7892 & 0.01691 \\
\hline 250.9766 & 0.5 & 250.9124 & 0.01694 & 250.9598 & 0.01761 & 250.9801 & 0.01695 \\
\hline
\end{tabular}

In table 1 . The frequency was deternmined by $f_{0}=250+\tilde{\delta} \cdot \Delta f$, where $\Delta f$ represented the frequency resolution ( $\Delta f=f_{s} / N$ ), $\tilde{\delta}$ is located in $[-0.5,+0.5]$. The frequency $f_{0}$ varies between $249.0234 \mathrm{~Hz}$ and $250.9766 \mathrm{~Hz}$ with a step of $1.953125 \mathrm{~Hz}$. The SNR is $9 \mathrm{~dB}$ and the $\sigma_{C R B}$ is about 
$0.0169(\mathrm{~Hz})$. in the Rife's method, when the $\tilde{\delta}$ is close to zero, incorrect polarity estimation (IPE) could lead to inproper estimation, however, which can almost be completely avoided in the proposed algorithm. Comparing with the Rife's method and the L\&Z\&M’s algorithm, the proposed algorithm has better performance.

\section{Conclusion}

This paper proposed an iterative DFT interpolation algorithm without weighting window that can accurately estimate frequency in a discrete spectrum. Simulation results show that the proposed algorithm is robust against mistaken location of the spectral line, and has good performance when the SNR is above zero. In future research, we will try to eliminate noise to further improve the performance when the SNR is below zero.

\section{References}

[1]. D.C. Rife, G. Vincent, Use of the discrete Fourier transform in the measurement of frequencies and levels of tones, Bell Syst. Tech. J. 49 (1970) 197-228.

[2]. V.K. Jain, W.L. Collins, D.C. Davis, High-accuracy analog measurements via in-terpolated FFT, IEEE Trans. Instrum. Meas. 28 (1979) 113-122.

[3]. T. Grandke, Interpolation algorithms for discrete Fourier transforms of weighted signals, IEEE Trans. Instrum. Meas. 32 (1983) 350-355.

[4]. C. Offelli, D. Petri, Interpolation techniques for real-time multifrequency wave-form analysis, IEEE Trans. Instrum. Meas. 39 (1990) 106-111.

[5]. M. Xie, K. Ding, Corrections for frequency, amplitude and phase in a fast Fourier transform of a harmonic signal, Mech. Syst. Signal Process. 10 (1996) 211-221.

[6]. B. Quinn, Frequency estimation using tapered data, in: Proceedings of IEEE International Conference on Acoustics, Speech and Signal Processing, ICASSP 2006, IEEE, 2006, pp.73-76.

[7] .E. Aboutanios, Generalised DFT-based estimators of the frequency of a complex exponential in noise, in: 2010 3rd International Congress on Image and Signal Processing, CISP, IEEE, 2010, pp.2998-3002.

[8] .E. Aboutanios, Estimation of the frequency and decay factor of a decaying exponential in noise, IEEE Trans. Signal Process. 58 (2010) 501-509.

[9]. E. Aboutanios, Estimating the parameters of sinusoids and decaying sinusoids in noise, IEEE Instrum. Meas. Mag. 14 (2011) 8-14.

[10]. K. Duda, DFT interpolation algorithm for Kaiser-Bessel and Dolph-Chebyshev windows, IEEE Trans. Instrum. Meas. 60 (2011) 784-790.

[11]. Jiufei Luo, Zhijiang Xie, Ming Xie, Frequency estimation of the weighted real tones or resolved multiple tones by iterative interpolation DFT algorithm. Digital Signal Processing 41 (2015) 118-129.

[12]. D. Rife, R.R. Boorstyn, Single tone parameter estimation from discrete-time observations, IEEE Trans. Inf. Theory 20 (1974) 591-598. 